\title{
Antiretroviral concentration measurements as an additional tool to manage virologic failure in resource limited settings: a case control study
}

\author{
Allan Buzibye ${ }^{1 *}$, Joseph Musaazi ${ }^{1}$, Amrei von Braun², Sarah Nanzigu ${ }^{3}$, Christine Sekaggya-Wiltshire ${ }^{1}$, \\ Andrew Kambugu', Jan Fehr ${ }^{4}$, Mohammed Lamorde ${ }^{1}$, Ursula Gutteck ${ }^{5}$, Daniel Muller ${ }^{5}$, Stefanie Sowinski ${ }^{6}$, \\ Steven J. Reynolds ${ }^{7,8}$ and Barbara Castelnuovo ${ }^{1}$
}

\begin{abstract}
Background: Several studies demonstrate a correlation between sub-therapeutic concentrations of antiretroviral drugs and virologic failure. We examined the sensitivity, specificity and predictive values of sub-therapeutic drug levels in predicting viralogic failure.

Methods: This was a case control study with cases being samples of participants with virologic failure, and controls samples of participants with virologic suppression. We analyzed samples obtained from participants that had been on antiretroviral treatment (ART) for at least 6 months. Virologic failure was defined as HIV-RNA viral load $\geq 1000$ copies/ $\mathrm{ml}$. Sub-therapeutic drug levels were defined according to published reference cutoffs. The diagnostic validity of drug levels for virologic failure was assessed using plasma viral loads as a gold standard.

Results: Sub-therapeutic ART concentrations explained only $38.2 \%$ of virologic failure with a probability of experiencing virologic failure of 0.66 in a patient with low drug levels versus 0.25 for participants with measurements within or above the normal range. Approximately $90 \%$ of participants with ART concentrations above the lower clinical cut off did not have virologic failure.
\end{abstract}

Conclusions: These results support prior indication for therapeutic drug monitoring in cases of suspected virologic failure.

Keywords: Virologic failure, Therapeutic drug monitoring, Uganda, Resource limited setting, HIV

\section{Background}

Antiretroviral therapy (ART) has reduced morbidity and mortality in people living with HIV $[1,2]$. Currently, the World Health Organization recommends periodic viral load testing for monitoring the effectiveness of ART [3]. Virologic failure is defined according to the $2016 \mathrm{WHO}$ guidelines as two consecutive HIV-RNA viral loads $\geq 1000$ copies/ml [3]. Patients with an initial detectable viral load test result receive intensive adherence counselling sessions before the viral load test is repeated, in order

\footnotetext{
*Correspondence: abuzibye@idi.co.ug

${ }^{1}$ Infectious Diseases Institute, Makerere University, College of Health

Sciences, P. O. BOX 22418, Kampala, Uganda

Full list of author information is available at the end of the article
}

to differentiate non-adherence to ART from virologic failure due to drug resistance. When the repeat viral load result remains $\geq 1000$ copies $/ \mathrm{ml}$ patients are switched to another regimen.

Two factors that may contribute to virologic failure include pre-treatment resistance and acquired drug resistance $[4,5]$. Acquired drug resistance normally results from sub-therapeutic drug exposure. Factors that may lead to sub-therapeutic exposure include single nucleotide polymorphisms $[4,5]$, drug-drug interactions, drug-herb interactions, non-adherence and mal-absorption [6-9]. Therapeutic drug monitoring (TDM) may detect suboptimal concentrations, and therefore inform the clinicians on the need to address factors which may 
lead to drug resistance and prevent unnecessary switching where treatment options are already limited.

Therapeutic drug monitoring is the clinical practice of measuring specific drug levels at designated intervals with the purpose of maintaining a constant blood concentration and optimize dosage for individual patients [10]. Among the antiretroviral drug classes, non-nucleoside reverse transcriptase inhibitors (NNRTIs) and protease inhibitors (PIs) meet the proposed criteria for TDM such as the presence of a dose-response relationship [11]. Several platforms are employed for TDM of HIV drugs [12-14]. In resource-limited settings, high performance liquid chromatography with ultraviolet detection (HPLC-UV) is commonly used. We investigated the sensitivity, specificity and predictive values of NNRTIs and PIs drug levels in predicting virologic failure.

\section{Materials and methods}

This study was conducted at the Infectious Diseases Institute (IDI), Makerere University, an HIV centre of excellence located in Mulago teaching Hospital in Kampala with over 8000 HIV-positive individuals receiving care [15]. The IDI clinic began providing HIV care in 2002, while free ART has been provided since April 2004.

This was a case control study. For our analysis we used samples from patients enrolled in the "Resistance in HIVinfected adults in North and South" (RHINOS) study [16]. The RHINOS study was conducted between June and September 2015 at IDI, and enrolled patients on any first- or second-line ART regimen for at least 6 months. The RHINOS study offered viral load testing to all participants and resistance testing to those with $\mathrm{VL}>1000$ copies/ml [16].

Cases were defined as participants with virologic failure $(\mathrm{VL}>1000$ copies/ml), while controls were patients with a measurement of $\mathrm{VL} \leq 1000$ copies. Controls were matched on age and gender at the ratio of $1: 2$, and samples were obtained using the cumulative sampling technique.

An in-house multiplex assay high performance liquid chromatography with ultraviolet detection (HPLCUV) was developed and validated to measure the serum concentration of efavirenz, nevirapine, atazanavir and lopinavir. The HPLC UV system used was a Shimadzu LC-2010HT with an inbuilt auto sampler, pumps, and UV detector (Shimadzu, Kyoto, Japan), controlled by CLASS-VP software version 6.1 (Shimadzu, Kyoto, Japan). The analytical phenyl hexyl column was a Betasil $150 \times 3 \mathrm{~mm}, 5 \mu \mathrm{m}$ (Thermo Scientific, Waltham, USA) protected by an inline filter. Sample processing involved protein precipitation from serum. Dual detection was achieved at 210 and $254 \mathrm{~nm}$. The mobile phase consisted of $50 \mathrm{mM}$ ammonium acetate/0.1 mmol/l EDTA/0.1\% formic acid and acetonitrile/water $(9 / 1 \mathrm{v} / \mathrm{v})$. The flow rate was set at $0.45 \mathrm{ml} / \mathrm{min}$ with gradient elution. The method was validated over a concentration range of $1-15 \mathrm{mg} / \mathrm{l}$ for nevirapine, efavirenz, lopinavir and $0.2-3 \mathrm{mg} / \mathrm{l}$ for atazanavir. The assay was accurate (98.0-109.6\%) with inter and intra-day coefficient of variation less than $11 \%$. Drug levels were considered sub-therapeutic if they were below the lower clinical cut-off [17-19].

Serum concentration data were captured using EpiData version 3.1 (EpiData association, Odense, Denmark). Serum data were merged with other data collected from the main RHINOS study dataset and analyzed using STATA/IC version 14.2 (StataCorp, College Station, Texas 77845 USA). McNemar's Chi-square test was used to compare categorical characteristics across cases and controls, such as WHO stage. Mann-Whitney Wilcoxon rank-sum test was used for continuous variables, like CD4 counts, and serum drug concentrations. Sensitivity, specificity and predictive values (PV) of drug levels in predicting virologic failure was estimated. Receiver operating characteristic (ROC) curve analysis was used to define a cut-off that would provide a maximum value for specificity and sensitivity.

\section{Results}

Of 2511 patients enrolled in RHINOS, 198 (7.9\%) patients had VL $>1000$ copies/ml, and were eligible as cases; two cases were excluded because they had no matching controls, and five other cases were excluded because samples could not be identified. Therefore, 191 cases and 382 controls for a total of 573 samples were analyzed for serum drug levels.

Table 1 compares the baseline characteristics of the cases and the controls. The median CD4 count was lower in the cases $(262$ cells $/ \mu \mathrm{l}$,) compared to the controls $(500$ cells $/ \mu \mathrm{l}$, $)(\mathrm{P}$-value $<0.01)$. Cases had a higher proportion of participants in WHO 3 or 4 stage, compared to controls $(61.8 \%$ versus $51.2 \%$, $\mathrm{P}$-value $=0.02)$. We observed a higher proportion of cases on PI-based ART regimen compared to controls (25.7\% versus $11.8 \%$, P-value $<0.01)$. Cases were matched with controls by gender and age, and therefore, baseline differences were mainly observed in WHO staging and CD4 counts. The average time from the last intake of a dose of ART was $8 \mathrm{~h}$.

For nevirapine, efavirenz and atazanavir, mean and the median drug concentrations were significantly lower in the cases compared to controls. For lopinavir a trend towards lower concentrations was noted in cases (Table 2). Most drug levels were within the published clinical ranges for all the drugs (Fig. 1).

Overall, TDM had a sensitivity of $38.2 \%$ and a positive predictive value (PPV) of $65.8 \%$ for virologic failure. 
Table 1 Patients' characteristics by cases and controls

\begin{tabular}{|c|c|c|c|}
\hline \multirow[t]{2}{*}{ Characteristics } & $\begin{array}{l}\text { Cases }(\mathrm{VL}>1000)(\text { copies } / \mu \mathrm{l}) \\
\mathrm{N}=191\end{array}$ & $\begin{array}{l}\text { Controls }(\mathrm{VL} \leq 1000) \text { (copies/ul) } \\
\mathrm{N}=382\end{array}$ & \multirow[t]{2}{*}{ P-value } \\
\hline & $\mathrm{N}(\%)$ & $\mathrm{N}(\%)$ & \\
\hline \multicolumn{4}{|l|}{ Gender, number (\%) } \\
\hline Male & $58(30.4)$ & $116(30.4)$ & - \\
\hline Female & $133(69.6)$ & $266(69.6)$ & - \\
\hline Age in years, median (IQR) & $37(29-43)$ & $37(29-43)$ & - \\
\hline $18-34$ & $75(39.3)$ & $150(39.3)$ & - \\
\hline$\geq 35$ & $116(60.7)$ & $232(60.7)$ & - \\
\hline \multicolumn{4}{|l|}{ WHO stage, number (\%) } \\
\hline 1 or 2 & $73(38.2)$ & $186(48.8)$ & \multirow[t]{2}{*}{0.02} \\
\hline 3 or 4 & $118(61.8)$ & $195(51.2)$ & \\
\hline CD4 cell counts per $\mu \mathrm{l}$, median (IQR) & $262(118-429)$ & $500(346-661)$ & $<0.01$ \\
\hline Duration on ART in months, median (IQR) & $47(27-82)$ & $46(27-93)$ & 0.22 \\
\hline Time since HIV diagnosis in years Median (IQR) & $7(3-11)$ & $8(4-11)$ & 0.30 \\
\hline ART_NNRT (NVP, EFV), number (\%) & $141(74.2)$ & $339(88.7)$ & - \\
\hline ART_PI (ATV, LPV), number (\%) & $49(25.7)$ & $45(11.8)$ & $<0.01$ \\
\hline
\end{tabular}

Gender and age were used as matching variables, so P-values on them is not relevant

$N$ number, IQR interquartile range, VL viral load, WHO World Health Organization, ART antiretroviral therapy, ATV atazanavir, LPV lopinavir, EFV efavirenz, NVP nevirapine, NNRTI nonnucleoside reverse transcriptase inhibitor, $P /$ protease inhibitor

Table 2 Distribution of drug concentrations by cases and controls

\begin{tabular}{llllllll}
\hline Drug & \multicolumn{2}{l}{ Cases } & & \multicolumn{2}{l}{ Controls } & \multirow{2}{*}{ P-value* } \\
\cline { 2 - 3 } & N & Median (IQR) & & N & Median (IQR) & \\
\hline Nevirapine & 65 & $5.37(2.81-7.64)$ & & 116 & $8.18(6.38-12.69)$ & $<0.01$ \\
Efavirenz & 77 & $1.60(0.00-2.50)$ & & 221 & $2.72(1.78-4.71)$ & $<0.01$ \\
Lopinavir & 34 & $6.41(0.00-10.70)$ & & 24 & $9.84(5.54-13.57)$ & 0.06 \\
Atazanavir & 15 & $0.00(0.00-1.44)$ & & 21 & $2.50(1.29-3.24)$ & 0.01 \\
\hline
\end{tabular}

*P-values comparing median drug concentrations between cases and controls using Mann-Whitney Wilcoxon rank-sum test. Significant differences were observed in concentrations of nevirapine, efavirenz and atazanavir

Sensitivity, specificity, PPV and negative predictive value (NPV) respectively for each drug are shown in Table 3. Using ROC curve analysis, for nevirapine, a cut off of $7.8 \mathrm{mg} / \mathrm{l}$ resulted in a sensitivity of $78 \%$ and a specificity of $56 \%$ (AUC-0.71). For efavirenz, a cut off of $2.7 \mathrm{mg} / \mathrm{l}$ resulted in a sensitivity of $64 \%$ and a specificity of $54 \%$ (AUC-0.61). Sample size was inadequate to establish a cut-off for atazanavir. No calculation was performed for lopinavir as there was no significant difference in concentrations between cases and controls.

\section{Discussion}

Previous studies have shown a positive relationship between drug levels and virologic failure [19-22]. However, to our knowledge this is the first sub-Saharan Africa study to explore the sensitivity, specificity and predictive

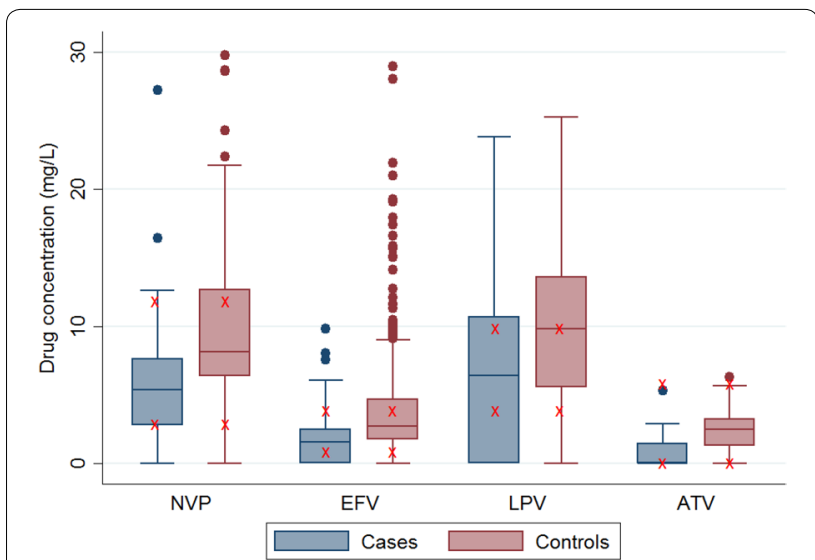

Fig. 1 A box plot of drug concentration by drug type stratified by cases and controls. $\mathrm{x}=$ Marks the lower and upper cut-off of normal ranges

values of a panel of non-nucleoside reverse transcriptase inhibitors and protease inhibitors for predicting virologic failure. Serum concentrations of ART among cases were significantly lower than concentrations among controls suggesting that low drug levels contribute to virologic failure. We show that an approach using TDM as a screening tool would identify approximately one third of the cases of virologic failure in our study population.

A patient with a low level of ART was more likely to be experiencing virologic failure compared to a patient 
Table 3 SE, SP, PPV and NPV for TDM methods for detecting virologic failure for different ARV drugs

\begin{tabular}{lllll}
\hline Drug & SE $(\mathbf{9 5} \% \mathbf{C l})$ & SP $(\mathbf{9 5} \% \mathbf{C l})$ & PPV $\mathbf{( 9 5 \% ~ C l )}$ & NPV (95\% Cl) \\
\hline Nevirapine & $27.7(16.8-38.6)$ & $94.0(89.6-98.3)$ & $72.0(54.4-89.6)$ & $69.9(62.7-77.1)$ \\
Efavirenz & $41.6(30.6-52.6)$ & $89.1(85.0-93.2)$ & $57.1(44.2-70.1)$ & $81.4(76.5-86.3)$ \\
Lopinavir & $44.1(27.4-60.8)$ & $83.3(68.4-98.2)$ & $78.9(60.6-97.3)$ & $51.3(35.6-67.0)$ \\
Atazanavir & $53.3(28.1-78.6)$ & $85.7(70.7-99.0)$ & $72.7(46.4-99.0)$ & $72.0(54.4-89.6)$ \\
Overall & $38.2(31.3-45.1)$ & $90.1(87.1-93.1)$ & $65.8(56.9-74.6)$ & $74.5(70.5-78.4)$ \\
\hline
\end{tabular}

SE sensitivity, SP specificity, PPV positive predictive value, NPV negative predictive value

with higher concentrations. Generally, the predictive values were $70 \%$ or higher except for efavirenz whose PPV was $57.1 \%$ and lopinavir whose NPV was $51.3 \%$. Here we report a higher PPV for efavirenz compared to what was reported earlier by Catia and colleagues (50\%) [19] probably due to a higher prevalence of virologic failure in our setting compared to the Swiss setting. Our study identified greater sensitivity and specificity when using lower cutoffs for nevirapine $(7.8 \mathrm{mg} / \mathrm{l})$ and efavirenz $(2.7 \mathrm{mg} / \mathrm{l})$ that were greater than previously published cutoffs (nevirapine $3 \mathrm{mg} / \mathrm{l}$ and efavirenz $(1 \mathrm{mg} / \mathrm{l})$. Our cut-offs are likely influenced by a greater proportion of participants with high concentrations of NNRTI (Fig. 1) that may be explained by slow metabolizer polymorphisms of cytochrome P450 2B6 that are more common in African settings [23].

Our results confirm previous findings about the performance of TDM in predicting virologic failure when compared with viral load measurements [24]. TDM has been reported to be a poor predictor of virologic failure compared to other adherence measures such as alectronic adherence monitoring device and pharmacy refill [24-26]. However, TDM performs far better than selfreported adherence. In a study done in Cameroon [27], virologic failure was associated with nevirapine concentration but not with self-reported adherence. The sensitivity, specificity, positive and negative predictive values were: $20.5 \%, 91.7 \%, 44.4 \%$ and $78.0 \%$ respectively when TDM was compared to viral loads. The respective values for self-reported adherence were $2.6 \%, 97.5 \%, 25.0 \%$ and $75.5 \%$.

No reliable data on adherence and time of drug intake was available to support interpretation of the results. It was not possible to determine if the low concentrations were a true reflection of lower steady state concentrations or because some patients had recently had a treatment interruption and the TDM sample was collected while they had not yet achieved steady-state concentration. Prospective studies with serial concentration measurements preceding a virologic outcome would yield additional information on the TDM approach. Our study was conducted in a clinical research facility, where TDM facilities and technical capacity are available and few centers are available for TDM in Africa. In contrast, viral load testing is more readily available in resource limited settings. If TDM were confirmed to provide additional value, feasibility and cost considerations for expansion of this test would need to be explored.

\section{Conclusion}

In agreement with previous studies, this study suggests that low drug levels of ART could contribute to virologic failure. Prospective studies in resource limited settings are needed to investigate this relationship.

\footnotetext{
Abbreviations

ART: antiretroviral therapy; HIV: human immunodeficiency virus; RNA: ribonucleic acid; WHO: World Health Organization; TDM: therapeutic drug monitoring; NNRTIs: nonnucleotide reverse transcriptase inhibitors; P.Is: protease inhibitors; EFV: efavirenz; NVP: nevirapine; LPV: lopinavir; ATV: atazanavir; HPLC-UV: high performance liquid chromatography with ultraviolet detection; VL: viral load; VF: virologic failure; IDI: Infectious Diseases Institute; RHINOS: resistance in HIV-infected adults in North and South; IQR: interquartile range; NVP: negative predictive value; PPV: positive predictive value; SE: sensitivity; SP: specificity; PK: pharmacokinetics; ROC: receiver operating characteristic.
}

\section{Acknowledgements}

Technical support and reference substances were offered by the Department of Clinical Chemistry, University Hospital, University of Zurich. Tuition support was provided by Researchers for Global Health (R4GH).

\section{Authors' contributions}

AB-principle investigator, determined drug concentrations, writing manuscript and data collections; JM-data management and statistical analysis; AvB, CS and BC-collected data in the previous study, proposal writing and manuscript writing; ML, SN and SJR-manuscript writing; SS, AK and JF-proposal writing; UG and DM-technical support during drug concentration measurements. All authors read and approved the final manuscript.

\section{Funding}

This research was supported by a Grant from the National Institutes of Health $(\mathrm{NIH})$, University of California, San Francisco-Gladstone Institute of Virology \& Immunology Center for AIDS Research, P30-AI027763 and in part (SJR) by the Division of Intramural Research, NIAID/NIH. Support for manuscript writing was provided by Fogarty International Center, National Institutes for Health (Grant \#D43TW009771 "HIV co-infections in Uganda: TB, Cryptococcus, and Viral Hepatitis").

\section{Availability of data and materials}

The data that support the findings of this study are available from the RHINOS study team but restrictions apply to availability of these data and so not publicly available. Data are however available from corresponding author upon reasonable request and with permission of the RHINOS study team. 


\section{Ethical approval and consent to participate}

Approval was obtained from the Institutional Review Board (IRB) of Makerere University (Approval number: SBS-HDREC-375), and the Uganda National Council for Science and Technology to use the stored samples for this study. Written consent was obtained from all study participants of the RHINOS study.

\section{Consent for publication}

Not applicable.

\section{Competing interests}

The authors declare that they have no competing interests.

\section{Author details}

${ }^{1}$ Infectious Diseases Institute, Makerere University, College of Health Sciences, P. O. BOX 22418, Kampala, Uganda. ${ }^{2}$ Division of Infectious Diseases and Tropical Medicine, University Hospital of Leipzig, University of Leipzig, Leipzig, Germany. ${ }^{3}$ Department of Pharmacology, Makerere University, College of Health Sciences, Kampala, Uganda. ${ }^{4}$ Department of Public Health, University Hospital, University of Zurich, Zurich, Switzerland. ${ }^{5}$ Department of Clinical Chemistry, University Hospital, University of Zurich, Zurich, Switzerland. ${ }^{6}$ The Gladstone Institute of Virology and Immunology, University of California, San Francisco, San Francisco, CA, USA. 7 Division of Intramural Research, National Institute of Allergy and Infectious Diseases, National Institutes of Health, Bethesda, MD, USA. ${ }^{8}$ Johns Hopkins University School of Medicine, Baltimore, MD, USA

\section{Received: 2 May 2019 Accepted: 27 November 2019}

Published online: 06 December 2019

\section{References}

1. Murphy EL, Collier AC, Kalish LA, Assmann SF, Para MF, Flanigan TP, et al. Highly active antiretroviral therapy decreases mortality and morbidity in patients with advanced HIV disease. Ann Intern Med. 2001;135(1):17-26.

2. Miiro G, Todd J, Mpendo J, Watera C, Munderi P, Nakubulwa S, et al. Reduced morbidity and mortality in the first year after initiating highly active anti-retroviral therapy (HAART) among Ugandan adults. Trop Med Int Health. 2009;14(5):556-63.

3. WHO Guidelines Approved by the Guidelines Review Committee. In: nd, editor. Consolidated guidelines on the use of antiretroviral drugs for treating and preventing HIV infection: recommendations for a Public health approach. Geneva: World Health Organization. Copyright (c) World Health Organization 2016.; 2016.

4. Hamers RL, Schuurman R, Sigaloff KC, Wallis CL, Kityo C, Siwale M, et al. Effect of pretreatment HIV-1 drug resistance on immunological, virological, and drug-resistance outcomes of first-line antiretroviral treatment in sub-Saharan Africa: a multicentre cohort study. Lancet Infect Dis. 2012;12(4):307-17.

5. Hassan AS, Nabwera HM, Mwaringa SM, Obonyo CA, Sanders EJ, Rinke de Wit TF, et al. HIV-1 virologic failure and acquired drug resistance among first-line antiretroviral experienced adults at a rural HIV clinic in coastal Kenya: a cross-sectional study. AIDS Res Ther. 2014;11(1):9.

6. Bulage L, Ssewanyana I, Nankabirwa V, Nsubuga F, Kihembo C, Pande G, et al. Factors associated with virological non-suppression among HIVpositive patients on antiretroviral therapy in Uganda, August 2014-July 2015. BMC Infect Dis. 2017;17(1):326.

7. de Maat MM, Huitema AD, Mulder JW, Meenhorst PL, van Gorp EC, Mairuhu AT, et al. Subtherapeutic antiretroviral plasma concentrations in routine clinical outpatient HIV care. Ther Drug Monit. 2003;25(3):367-73.

8. Hawkins C, Ulenga N, Liu E, Aboud S, Mugusi F, Chalamilla G, et al. HIV virological failure and drug resistance in a cohort of Tanzanian HIVinfected adults. J Antimicrob Chemother. 2016:71(7):1966-74.

9. von Braun A, Castelnuovo B, Ledergerber B, Cusato J, Buzibye A, Kambugu $A$, et al. High efavirenz serum concentrations in TB/HIV-coinfected Ugandan adults with a CYP2B6 516 TT genotype on anti-TB treatment. J Antimicrob Chemother. 2019;74(1):135-8.

10. Kang JS, Lee MH. Overview of therapeutic drug monitoring. Korean J Intern Med. 2009;24(1):1-10.

11. Ribera E, Lopez-Cortes LF, Soriano V, Casado JL, Mallolas J. Therapeutic drug monitoring and the inhibitory quotient of antiretroviral drugs: can they be applied to the current situation? Enferm Infecc Microbiol Clin. 2005;23(Suppl 2):41-67.
12. Bollen PDJ, de Graaff-Teulen MJA, Schalkwijk S, van Erp NP, Burger DM. Development and validation of an UPLC-MS/MS bioanalytical method for simultaneous quantification of the antiretroviral drugs dolutegravir, elvitegravir, raltegravir, nevirapine and etravirine in human plasma. J Chromatogr B Analyt Technol Biomed Life Sci. 2019;1105:76-84.

13. Charbe N, Baldelli S, Cozzi V, Castoldi S, Cattaneo D, Clementi E. Development of an HPLC-UV assay method for the simultaneous quantification of nine antiretroviral agents in the plasma of HIV-infected patients. J Pharm Anal. 2016:6(6):396-403.

14. Patel SH, Ismaiel OA, Mylott WR Jr, Yuan M, Hauser KF, McRae M. Simultaneous determination of intracellular concentrations of tenofovir, emtricitabine, and dolutegravir in human brain microvascular endothelial cells using liquid chromatography-tandem mass spectrometry (LC-MS/ MS). Anal Chim Acta. 2019;1056:79-87.

15. Nwaka S, Ochem A, Besson D, Ramirez B, Fakorede F, Botros S, et al. Analysis of pan-African Centres of excellence in health innovation highlights opportunities and challenges for local innovation and financing in the continent. BMC Int Health Hum Rights. 2012;12:11.

16. von Braun A, Sekaggya-Wiltshire C, Bachmann N, Ssemwanga D, Scherrer AU, Nanyonjo $\mathrm{M}$, et al. HIV-1 drug resistance among Ugandan adults attending an urban out-patient clinic. J Acquir Immune Defic Syndr (1999). 2018;78(5):566-73.

17. Breilh D, Pellegrin I, Rouzes A, Berthoin K, Xuereb F, Budzinski H, et al. Virological, intracellular and plasma pharmacological parameters predicting response to lopinavir/ritonavir (KALEPHAR study). AIDS. 2004;18(9):1305-10.

18. Higgins N, Tseng A, Sheehan NL, la Porte CJ. Antiretroviral therapeutic drug monitoring in Canada: current status and recommendations for clinical practice. Can J Hosp Pharm. 2009;62(6):500-9.

19. Marzolini C, Telenti A, Decosterd LA, Greub G, Biollaz J, Buclin T. Efavirenz plasma levels can predict treatment failure and central nervous system side effects in HIV-1-infected patients. AIDS. 2001;15(1):71-5.

20. Alexander CS, Asselin JJ, Ting LS, Montaner JS, Hogg RS, Yip B, et al. Antiretroviral concentrations in untimed plasma samples predict therapy outcome in a population with advanced disease. J Infect Dis. 2003;188(4):541-8.

21. Court R, Gordon M, Cohen K, Stewart A, Gosnell B, Wiesner L, et al. Random lopinavir concentrations predict resistance on lopinavir-based antiretroviral therapy. Int J Antimicrob Agents. 2016;48(2):158-62.

22. Kimulwo MJ, Okendo J, Aman RA, Ogutu BR, Kokwaro GO, Ochieng DJ, et al. Plasma nevirapine concentrations predict virological and adherence failure in Kenyan HIV-1 infected patients with extensive antiretroviral treatment exposure. PLoS ONE. 2017;12(2):e0172960.

23. Mukonzo JK, Owen JS, Ogwal-Okeng J, Kuteesa RB, Nanzigu S, Sewankambo N, et al. Pharmacogenetic-based efavirenz dose modification: suggestions for an African population and the different CYP2B6 genotypes. PLoS ONE. 2014;9(1):e86919.

24. Orrell C, Cohen K, Leisegang R, Bangsberg DR, Wood R, Maartens G. Comparison of six methods to estimate adherence in an ART-naive cohort in a resource-poor setting: which best predicts virological and resistance outcomes? AIDS Res Ther. 2017;14(1):20.

25. Deschamps AE, De Geest S, Vandamme AM, Bobbaers H, Peetermans WE, Van Wijngaerden E. Diagnostic value of different adherence measures using electronic monitoring and virologic failure as reference standards. AIDS Patient Care STDs. 2008;22(9):735-43.

26. San Lio MM, Carbini R, Germano P, Guidotti G, Mancinelli S, Magid NA, et al. Evaluating adherence to highly active antiretroviral therapy with use of pill counts and viral load measurement in the drug resources enhancement against AIDS and malnutrition program in Mozambique. Clin Infect Dis. 2008:46(10):1609-16.

27. Kouanfack C, Laurent C, Peytavin G, Ciaffi L, Ngolle M, Nkene YM, et al. Adherence to antiretroviral therapy assessed by drug level monitoring and self-report in cameroon. J Acquir Immune Defic Syndr. 2008:48(2):216-9.

\section{Publisher's Note}

Springer Nature remains neutral with regard to jurisdictional claims in published maps and institutional affiliations. 